It should be noted that Lemma 1 is interesting, independent of strong Lie ideals, for it generalizes a result of [1]. This generalization can be stated as follows:

THEOREM 2. Let $A$ be a simple ring of characteristic $\neq 2$, with either its center $Z=(0)$ or of dimension greater than 16 over its center, and with an involution defined on it; then if either $K, S,[K, K]$ or $[K, S]$ are finite dimensional, $A$ is finite dimensional.

\title{
REFERENCES
}

1. W. E. Baxter, Lie simplicity of a special class of associative rings. II, Trans. Amer. Math. Soc. vol. 87 (1958) pp. 63-75.

2. I. N. Herstein, Lie and Jordan systems in simple rings with involution, Amer. J. Math. vol. 78 (1956) pp. 629-649.

University of Delaware

\section{TRIANGLE INEQUALITY IN $l$-GROUPS}

\section{J. A. KALMAN}

In $[1$, p. 309] G. Birkhoff remarks that "In a commutative l-group, we can ... prove the triangle inequality $|a+b| \leqq|a|+|b|$, but this does not seem to hold in general." The purpose of this note is to show that in fact if

$$
|a+b| \leqq|a|+|b|
$$

for all $a$ and $b$ in the additive l-group $G$, then $G$ is commutative.

Proof. It is sufficient to show that any two positive elements of $G$ are permutable [2, p. 234]. Suppose therefore that $x$ and $y$ are positive elements of $G$. Taking $a=-x$ and $b=-y$ in (1), we obtain $x+y$ $\geqq|-x-y|=y+x$. Similarly $y+x \geqq x+y$. Hence $x+y=y+x$. This completes the proof.

\section{REFERENCES}

1. G. Birkhoff, Lattice-ordered groups, Ann. of Math. vol. 43 (1942) pp. 298-331.

2. - Lattice theory, Amer. Math. Soc. Colloquium Publications, rev. ed., vol. 25, New York, 1948.

UNIVERSITY OF AUCKLAND

Received by the editors August 1, 1959. 